\title{
\begin{tabular}{l|l} 
pcori)? & PATIENT-CENTERED OUTCOMES RESEARCH INSTITUTE \\
RESEARCH SUMMARY
\end{tabular}
}

\section{Comparing Online and In-Person Family Therapy for Teens with Traumatic Brain Injury and Their Parents}

Principal investigator

Shari L. Wade, PhD
Organization

Cincinnati Children's Hospital Medical Center

\section{What was the research about?}

Violent bumps or blows to the head can cause traumatic brain injury, or TBI. Teens may develop TBI from accidents or sports injuries. People with TBI often have headaches, nausea, dizziness, confusion, and mood changes. Some TBIs cause physical, emotional, and behavioral problems that may last a long time and that may be stressful for families. Family problemsolving therapy, or F-PST, teaches skills to help families communicate, manage anger, and adjust to changes in daily life.

In this study, the research team wanted to learn if online F-PST was similar to in-person F-PST in reducing the effects of TBI for teens and their parents. The team compared three ways of offering F-PST to teens and their parents:

- Online F-PST with a therapist: hour-long video calls with a therapist and online materials, including videos and ways to practice skills learned during the calls

- Online F-PST without a therapist: access to online materials that teens and parents could go through on their own; this group didn't meet with a therapist

- In-person F-PST: hour-long meetings with a therapist at a hospital or clinic and printed materials

\section{What were the results?}

In this study, the online and in-person F-PST options had similar results for teens and their parents. Comparing the different ways of offering F-PST, the teens in the three groups didn't differ in

- Behaviors, such as mood swings or outbursts of anger

- Ability to get things done

- Quality of life

- $\quad \mathrm{TBI}$ symptoms

- Depression

The study found no difference in parents' depression or ability to deal with stress.

\section{Who was in the study?}

The study included 150 teens ages 14 to 19 , treated at one of five TBI centers in Ohio and Colorado, and their parents. All teens had mild to severe TBI with lasting changes in behavior. Of these teens, 83 percent were white, 11 percent were African American, and 7 percent were another race or more than one race; 4 percent were Hispanic. The average age was 16 , and 64 percent were teenage boys.

\section{What did the research team do?}

The research team assigned teens and their parents, by chance, to get F-PST in one of the three ways. In 
each approach, families could attend up to 10 sessions about staying positive, solving problems, and controlling emotions. The team offered up to four more sessions on topics such as how to handle a crisis.

Teens and their parents filled out surveys at the start of the study and again six and nine months later. Surveys asked about teens' behaviors and ability to get things done. Surveys also included questions about quality of life, depression, and TBI symptoms.

Doctors, teens with TBI and their parents, and a web designer helped design and carry out the study.

\section{What were the limits of the study?}

After six months, 19 percent of teens and parents hadn't completed treatment. The number of people who didn't finish treatment was about the same in each group. On average, families completed only six sessions. Results might have been different if families attended more sessions. Problems with the website and with video calls may have kept teens and parents from viewing programs online or attending online therapy sessions.

Future research could test ways to help more people attend more sessions.

\section{How can people use the results?}

Doctors, teens with $\mathrm{TBI}$, and their parents can use these results when considering different options for therapy.

To learn more about this project, visit www.pcori.org/Wade203. 